\title{
Intertextualidad y literatura comparada
}

\author{
José Manuel Losada Gaya \\ Oxford University
}

$\mathrm{E}_{\mathrm{p}}$ ntre los principales procesos de nuestro inconsciente se encuentran el desplazamiento y la condensación, ampliamente estudiados por la crítica psicoanalítica. Si bien formaban parte del acervo cultural y pedagógico desde varios siglos antes de nuestra era. Kruszewski y Jakobson fueron quienes los introdujeron por vez primera en sus estudios de lingüística estructuralista bajo la denominación de metonimia y metáfora. Otro proceso fundamental es la sinécdoque, a menudo relegada a un segundo plano debido a cierta proclividad a confundirla con la metonimia: en determinadas ocasiones es difícil distinguir el grado.de relación entre el término propio y el término figurado. De ahíque, como señala Bemard Dupriez, no hayan faltado quienes consideren que la metonimia no es sino una sinécdoque doble o, más precisamente, en dos tiempos: el generalizador y el particularizador. No ofrece la misma dificultad la metáfora por cuanto en ella el paso de un sentido a otro tiene lugar a través de una operación personal basada en una impresión o interpretación íntimas. Cabe el riesgo de que el uso tienda a convertirla en cliché, pero la diferencia.con los otros procesos señalados, así como con la alegoría y la comparación es neta. Hasta el punto de que no hayan faltado quienes, como indicalavier del Prado a propósito de Breton, fundamenten en esta categoría la modernidad poética de un texto (1993, 244). Otro proceso, a veces consciente, a veces inconsciente, es el de la intertextualidad.

Intertextualidad concebida no ya como comparación, sino como lectura comparatista, esto es, la determinación de relaciones. Esta posibilidad metodológica interesa por muchos motivos, entre ellos, por uno que los comparatistas

O Poligraflas 2 (1997) $9 \cdot 34$

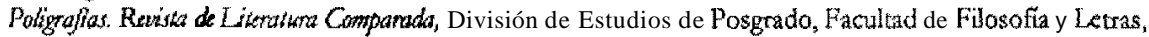
Universidad Nacional Autónoma de México, Ciudad Universitaria, México 04510, DI'.

Te! (525) 6221835(6\}. Fux (525) 622 t801; 6221826. E-mail pimentel@servidor.unammx 
hemos de remachar a tiempo y a destiempo: la literatura comparada no es comparar en el sentido del reduccionísmo comparatista; es algo mucho más fructífero, pero la simplificación a la que tienden quienes desconocen esta disciplina-facilidad en la que, sobre todo en sus comienzos, cayeron los mismos eomparatistas-será una amenaza contra la que habrá que luchar sin descanso. Es obvio que la terminología adoptada por los mundos anglosajón-Comparative Literature - gennarusta-Vergleichende Literaturwissenschafi-es mucho más ilustradora del trabajo que esta disciplina está llamada a ejercer en el mundo de la crítica literaria. En países como España, el error reduccionista proviene en no pocos casos del malhadado término «literatura comparada»-y otro tanto ocurre con la terminología adoptada por italianos (Letteratura comparata), portugueses (Literatura comparada), rumanos (Literatura comparata), ete.-traducción literal del término francés «littérature comparée,» debido a la avalancha de investigaciones que la crítica francesa aportó y continúa aportando en este terreno.

Quizá el acercamiento al texto en su carácter intertextual comenzara con la crítica semiótica de Julia Kristeva. Daniel-Henri Pageaux subraya el interés de la intertextualidad por cuanto esta categoría nos permite acometer un análisis y una lectura comparatistas a partir de un único texto. Éste, centro de la atenCÍón del estudioso, recibirá la denominación de «hipertexto» respecto del «hipotexto» que le precede en el tiempo. Dado que la literatura defiende como hecho incuestionable el «diálogo de culturas,» era lógico que se procediera a estudiar las consecuencias inmediatas de este principio. Entre ellas resalta que la absorción, transformación o remodelación de un texto precedente se puede considerar más bien como una intertextualidad en lugar de una intersubjetividad (Pageaux 1994, 18).1 En efecto, el comparatista sabe que este último término presupone la teona, últimamente tan denostada, de las influencias que un escritor o un texto ejercen sobre otro. De ahí que, tal y como indica el Dictionnaire de la théorie du langage de Greimas-Courtés, el concepto de intenextualidad haya suscitado gran interés por cuanto los procedimientos que implica parecen ofrecer una alternativa metodológica a la teoría de las influencias sobre la que venían basándose, por lo general, las investigaciones en el campo de la Literatura comparada. ${ }^{2}$

En los primeros vagidos de sus aprecíaciones sobre la lógica del texto. Julia Kristeva concebía la intertextualidad ora como el cruce de enunciados tomados a otros textos, ora como la transposición en la palabra comunicativa de enunciados anteriores o sincrónicos. Vengamos a la primera definici6n. No pasa desapercibido el acercamiento de cone estructuralista de la misma. Más precisamente, conviene recordar que, dentro de esta corriente. todo acto de enunciación viene a ser la producción nunegocéntrica de un conjunto semelfactivo de signos lingüísticos orientada según los siete polos tradicionales del esquema de la comunicación--ellocutor, el contacto, el destinatario, la situación, el contenido 
del mensaje o enunciado propiamente dicho, la lengua utilizada y la fonna estética que impregna el mensaje (Dupriez 1991, 182).

Partiendo de esta base y de la función que engloba a todos los enunciados en el texto, la profesora de origen búlgaro propone un acercamiento no ya meramente lingüístico sino translingüístico cuyo objetivo es «sustituir la antigua división retórica de los géneros por una tipología de los textos» (Kristeva 1978, 52).

Para lo cual es imprescindible recurrir a la noción de ideologema. Éste resulta de la verificación de una organización textual detenninada (o práctica semiótica) a partir de enunciados o secuencias que aquélla asimila o evoca dentro del espacio de los textos. No puede extrañar que, consiguientemente, el ideologema sea definido como la «función intertextual que se puede leer de forma materializada en los diferentes niveles de la estructura de cada texto y que se extiende en dicho texto proporcionándole sus coordenadas históricas y sociales» (53). La aplicación que la erudita hace sobre la novela permite conjeturarque las unidades lingüísticas son instrumentos aptos para establecer cada tipo de enunciado como una función. Por ende, dicha función viene a encadenarse a las restantes dentro de la totalidad de la producción textual. El paso siguiente tiene dos tiempos: en primer lugar la constitución de una tipología de los diferentes enunciados y en segundo lugar su origen extraliterario. En último término, cada tipo de texto podrá ser definido en su unidad, esto es, como ideologema (53-54).

De todo lo hasta aquí dicho podemos deducir tres ideas especialmente importantes: la problemática de la retórica de los géneros, la reflexión sobre el valor de las funciones del conjunto extraliterario dentro de un conjunto textual concreto y del acervo histórico que el ideologema aporta al texto en sí.

\section{.EI carácter nefasto de las extrapolaciones}

En su artículo titulado «En tomo al concepto de la intertextualidad,» José Antonio Millán Alba ve en este término una primera superación de los excesos inmanentistas (autotelismo, autarquismo) del estructuralismo literario. En dicho trabajo la intertextualidad se define como sigue: «presencia en un texto, de otro (u otros) ajenos al primero. pero que, sin embargo. resultan ser funcionales respecto de aquél; esto es, funcionan estructuralmente en su configuración estrictamente singular, constituyendo una de las hiladas de la urdimbre textual» $(1990,11)$. Esta definición apela, como su correlato, a otra que opera en el mismo campo: la transtextualidad, detenidamente estudiada por Genette en Palimpsesles. Como bien señala Millán Alba, el término de transtextualidad es especialmente interesante por cuanto en su relación con la intertextualidad conduce a la noción de historicidad: «el examen intrínseco de la pareja 'intertextualidad-lranstextualidad' nos aboca indefectiblemente. y al mismo tiempo lo recupera, al estudio de una 'historia' de la literatura.» De donde surge su interés por evitar el carácter 
autotélico que el estructuralísmo produjo en su tiempo: «La actual dicotomía entre historia de la literatura de un lado, y crítica literaria del otro, queda así resuelta mediante una de las funciones del texto que permite eltránsito natural del uno al otro campo, desde presupuestos, eso sí, estrictamente textuales 'que, aparentemente al menos ( , , ) resultan más rigurosos y fecundos que los que orientaron decisivamente la historia de la literatúra hasta los años cincuenta» (11-12),

Con otras palabras, es lo que Mikhail Bakhtine sostierie en los conceptos de dialogismo. polifonía y pluridiscursividad. El primero de éstos es interesante por una extensión globalizante que radica en las diferentes acepciones del diálogo. Abundando en esta última idea, el crítico ruso afirma: «El diálogo, en un sentido restringido, ciertamente no constituye más que una de las fortnas $(,,$.$) de la$ interacción verbal. Pero también es posible comprender la palabra 'diálogo' en un sentido amplío, es decir, no sólo como el intercambio en voz alta que implica a los individuos situados cara a cara, sino como todo tipo de intercambio verbal» (1977, 136).3 El dialogismo comprende en este autor dos tipos dë relaciones. La primera se da entre un texto determinado y otros, ya sean pasados o presentes. La segunda se puede observar entre un texto y el contexto sociocultural (seda por descontado la importancia que adquiere aquí la mimesis),

Ahora bien, entrar en el campo de la historia no quiere decir adoptar los presupuestos historicistas de corte positivista en su acepción peyorativa. MiUán Alba lo deja bien claro al esclarecer la otra vertiente de la intertextualidad: «esta definición tiene para nosotros la enorme ventaja de no sacarnos fuera del ámbito estrictamente literario.» Infiere con ello en el hecho evidente de que la dimensión intertextual és propiamente intrínseca al propio hecho textual: no puede sino ser «concebida como una función-entre otras-de la estructura literaria» $(1990,12)$. Procura así, y de hecho lo consigue, deslindarla de otros enfoques críticos extraliterarios: «No podemos compartir el enfoque de una historia de la literatura, y menos aún, de una literatura comparada, que encuentre su razón de ser no en ,su dimensión intrínseca (literatura), sino en la extrínseca (historia). A esta concepción es a la que tienden la historia de la literatura y comparatismo tradicionales en sus formulaciones positivistas, así como el enfoque filológico, acumulando datos. verificando biografías y ediciones, buscando parecidos, fuentes e influencias de acuerdo con el principio de analogía» (12). Algo semejante hace un gran comparatista rumano en un libro que tiene, entre otros, el mérito de ser el primer manual de literatura comparada en castellano. Al abordar la esencia de dicha disciplina. Alejandro Cioranescu alude a esta misma limitación: «La literatura comparada puede agregar datos y conocimientos nuevos. despenar y satis'facercuriosidades laterales $(, \ldots)$ pero no aportar un adarme para el conocimiento fundamental de la obra, considerada en su misterio y efectividad» (Millán Alba 1964). Todo esto es verdad, pero sólo relativamente. La raz6n es doble. 
En primer lugar, porque la intertextualidad no es una «función,» pero este error no es sino un desliz surgido como consecuencia del talante estructuralista que a menudo impregna las obras utilizadas por la mayoría de los críticos que han abordado la cuestión; además, Millán Alba había dado anteriormente una definici6n que no contenía este error. ${ }^{4}$ En segundo lugar, y esto ya es más grave, por cuanto la historia, el éomparatismo y la filología tradicionales ejercidas con rigor científico no quedan, ni mucho menos, al margen de la literatura (antes bien, muchasautodenominadas críticas literarias posteriores a los mencionados años cincuentas carecen de enfoques auténticamente literarios). Acumular datos, verificar biografías y ediciones, buscar parecidos, fuentes e influencias, recurrir al principio de la analogía (según Millán Alba), agregar conocimientos, despertar y satisfacercuriosidades laterales (según Cioranescu), san puntos de partida indispensables para la investigación litéraria. El malentendido está, como de costumbre, en los extremos: el fanatismo de los críticos tradicionalistas que se detienen en el historicismo no es menos perjudicial que el hermetismo de los críticos pseudomodemos que sobrepasan los límites de la interpretación.

Ni siquiera Billtine está al abrigo de estas extrapolaciones. Al dar por sentado que todo texto depende de un sistema y de un diálógo con otros textos-tanto desde un punto de vista formal como semántico-pragmático-elimina de raíz el carácter creativo de la literatura. Esto es ciertamente peligroso, pues propicia extrapolaciones que pueden llegara perder de vista el carácter propiamente literario del texto. Lo muestra la experiencia de buenos comentaristas como José Lean Machado, quienes, fascinados ante éstas teorías del crítico ruso, hacen suya la premisa de que «todo o discurso ( ... ) implica o outro, inscreve em si próprio o discurso do outro, porque a resposta tem como modelo outro ou autros discursos. Cada enunciado dialoga com outro enunciado, cada texto dialoga com outrós textos» $(1995,297)$. Pudiera aducirse que esto no es más que un desliz; no 10 creo. A na ser que admitamos una serie de deslices involuntarios. El mismo José Leon Machado, en su erudito estudio sobre la novela de Vergílio Ferreira, vuelve a caer en la misma trampa al comentar la célebre frase de Kristeva según la cual la intertextualidad es el cruce en un texto de enunciados tomados de otros textos. Llevando este postulado hasta sus últimas consecuencias, «cada texto é o lugar de encontro de outros textos, sejam eles anteriores ou coevos ao momento da escrita. Senda un texto um mosaico de citaçoes, a escrita toma-se então urna releitura do corpus já existente, urna actualização da memória» $(1995,309) .5$ Es una curiosa interpretaci6n del célebre aforismo de Persio en sus Sátiras: «De nihilo nihil.» No nos puede extrañar que José Lean Machado, siguiendo al pie de la letra los postulados de Bakhtine y Kristeva, deduzca que «Não se cria do nada, senao a partir de urna memória» (309). ¿Qué nos queda entonces de otras facultades del alma-inteligencia, voluntad, imaginaci6n-o de los sentidos', o 
de la más pura creatividad del ser humano? El hecho de que no haya literatura en vase dos, recluida en compartimientos estancos, no significa ni mucho menos que todo compartimiento se funda con los demás hasta llegar a confundirse en un magma informe e indiferenciado.

Este exceso, pura atrofia del hecho literario en sí, se observa con más nitidez aún en otro estudio de Bakhtine donde declara que el texto es la expresión de una conciencia que refleja algo. Cuando el texto se convierte en objeto de cognición, podernos hablar del reflejo de un reflejo-_du reflet d'un reflet"-(1984, 322). En esta línea, el reflejo adquiere el carácter de una 'señal la cual evoca a su vez otro texto, el cual se transmuta a su vez en señal y ésta ultima vuelve a evocar a otro tex.to, y así indefinidamente: el resultado sería pues una cadena que carece de principio positivamente conocido y cuyo final nunca llegará a conocerse. Si adoptamos la imagen acústica propuesta por Roland B arthes, es evidente que estas señales, dentro del espacio cerrado que es la literatura para toda esta corriente crítica, producen sin cesar no ya un hecho literario en sí, con vida e impulso propios, sino una sucesión de ecos en un medio clausurado: la cámara de ecos-"une chambre d'échos"-(Barthes 1975, 78). Todo esto ya lo había anunciado Berenson en 1948: "You canoot open your lips without being used as a mouthpiece by myriads upon myriads of the dead. You are Httle more then a disc recording the reproducing traditions that no awareness can fathom» (Aesthetics and History in the Visual Arts, citado por Broich 1989, 119).

«Reflejo de un reflejo,» «mosaico de citas,» «cámara de ecos,» «disco de grabación ... » Resonancia vacía donde el signo, y después el significado, han sido substituidos por la señal. ¿Qué nos queda de la literatura? Nada. Curiosamente, estamos ante un fenómeno sin precedentes: que el reducdonismo comparatista que se venía poniendo en tela de juicio sea llevado a cabo por los estructuralistas. Esta falacia debe ser puesta de manifiesto. Para ello es preciso denunciar la causa que la ha provocado; el estructuralismo llevado hasta sus últimas consecuencias. ${ }^{6}$ y el estructuralismo, cuando ha invadido el terreno propio de la literatura comparada, también la ha viciado de un inmanentismo sin posibilidad de resolución. Por ello estoy plenamente convencido de que el estructuralismo, por mucho que utilice tal o cual procedimiento, no saldrá del atolladero en el que se ha metido. Ni recurriendo a las nuevas teorías de la metáfora o la metonimia, ni sirviéndose de la intertextualidad, utilizada desde siempre pero con otro nombre. Esto no supone que el estructuralismo no pueda ayudamos a comprender mejor determinados aspectos de la literatura; ha dado un paso importante. Pero contiene desde el principio, como en germen, una serie de vicios epistemológicos de los que debe deshacerse, siempre y cuando quiera seguir aportando algo a la crítica literaria. Si no, morirá por inanición. Esto explica que tantos estudios no concluyan en algo concreto ni ofrezcan una bolsa de 
oxígeno. Si el objetivo que se marcan de antemano merece todos los elogios; no ocurre otro tanto con los resultados. No se entiende cómo la «pareja 'intertextualídad-transtextualidad' nos aboqu[e] indefectiblemente, y al mismo tiempo lo recuper[e], al estudio de una 'historia' de la literatura» (Míllán Alba 1990, 11) si esto se hace solamente mediante los métodos estructuralistas. Dicho de otra manera, la medicina que curará al estructuralismo no puede estar dentro del estructuralismo. Es preciso que ésta le venga desde fuera.

En un estudio titulado William Shakespeare, Víctor Hugo decía algo que puede aportarnos alguna luz al respecto. Hablando de la belleza que persiguen una y otra vez los espíritus literarios, declaraba que el primer rango siempre está libre y accesible. ${ }^{7}$ Para llegar a él es preciso separarse de todo lo que pueda desconcertar las audacias malsanas y cortar las alas del artista. Para Hugo, el impulso que estamos buscando no procede exclusivamente de lo que otros autores nos ofrecen, sino del arte mismo, de su inefabilidad, de su paradójico misterio, componente fundamental del hecho literario. Salta a la vista que lo importante, lo auténticamente importante, son la literatura y el autor literario. El texto literario ni se cierra indefinidamente en sí mismo ni depende exclusivamente de otros. Broich también desvela esta argucia en la que todos podemos caer si aceptamos el inmanentismo estructuralista: «lf a text, however, is intertextually connected with all previous texts, the post-structuralists concluded that there is no point in retaining the concept of the individual text of the individual author» $(1989,119)$. En estas lúcidas palabras ha salido a relucir un término-«post-estructuralismos-que ha llevado a su predecesor-el estructuralismo--a un callejón sin salida: En realidad, aquél no es más que una posterior extrapolación de éste, con la diferencia de que en éste )a tergiversación es tal que no puede sino ser más manifiesta todavía. Porque el análisis de las obras literarias específicas según los métodos post-e"structuralistas, sólo puede ser llevado a término forzando los textos literarios, con lo cual dejan de serlO: el crítico siempre será un crítico; puede hacer literatura, pero en su labor como crítico no puede arrogarse pretenciosamente-por muy bien que sepa disimularlo-el título de escritor literario. Y aquí lo está haciendo procurando ver por todas partes-reduccionismo literario--sucesión de intertextualidades. «If every text,» nos recuerda Broich, «is 'intertextual in all its elements, as the post-structuralísts assume, this means that the term 'intertextuality' cómpletely loses that distinguishing force which $\mathrm{aH}$ scholarly terms ougth lo have» (119).

Esta afirmación de Broich demuestra algo que, aun siendo evidente, muchos críticos no aceptan: en primer lugar, que están clausurando la literatura dentro de una epistemología inmanentista sin solución alguna; en segundo lugar, que el remedio que procuran utilizar también está minado en su base por el mismo inmanentismo: esto es, un redụccionismo comparatista de corte estructuralísta. 
La pescadilla se muerde la cola: pierden la capacidad de distinguir qué es lo específico de tal o cual texto y de talo cual autor: «lf the idea of the individual author has to be abandoned-como se deduce obligatoriamente de esta extrapolación ilegítima-the consequences would be that for instanee we cannot distinguish between a calculated employment of references to other texts within a literary text and mere influences of which the author himself may not have been aware ofwhich he may have been fighting against» (119-120).

Tomando el título del célebre libro de Harold Bloom, la ansiedad de la influencia que tantos críticos se empeñan en desvelar aun donde ésta no existe y, sobre todo, la simplificación de la literatura al reflejo de influencias, nos sugieren que hay una puerta por donde puede penetrar la luz en este pozo donde apenas se distingue quién es quién. Víctor Hugo firmaría sin dudar estapuerta a la esperanza que nos permite desembarazarnos de una atmósferararefacta. Negar que' cualquierautor posterior no pueda parangonarsé con los precedentes sería negar la fuerza creadora del intelecto humano y, consecuentemente, negar toda transcendencia. Ciertamente, la solución 'no es fácil, pero es la única: conocerse a sí mismo, saber identificarse, salir de la masa informe que engulle autores y obras sin detenerse en distingos: en lo que respecta al crítico, no ser masa, utilizar su propia capacidad de análisis y síntesis; en lo que respecta al autor, utilizar. en una medida proporcionada, 'los ecos que le envían otros textos adaptándolos a su propio tiempo, espacio y conciencia artística: ser otro. ${ }^{8}$

Respecto de la segunda definición de.Kristeva-la intertextualidad concebida como transposición en la palabra comunicativa de enunciados anteriores y sincrónicos--cabe también hacer una serie de aclaraciones. La primera es la que sugiere José Antonio Millán respecto de la evolución de la crítica en su utilización de esta categoría. Tiempo después de las afirmaciones contenidas en Sèméiotikè, Julia Kristeva proponía la substitución del término de intertextualidad por el de transposición, y ello. debido a «]a evidente manipulaci6n que [el primero] había venido sufriendo al ser considerado por algunos como la máscara de ese cajón de sastre que es la semi6tica había adoptado para decir 'lo de toda la vida'» (1990, 15). Salta a la vista la alusión, acertada, a la reprobación que las modernas metodologías comparatistas han hecho a los estudios tradicionalistas de literatura comparada.

No está de más recordar las detracciones de René Wellek contra un positivismo minado en su base y que él considera originario de la escuela francesa. Wellek denomina esta degeneración con el nombre de factualism y arguye poniendo en duda ese hecho interrnediario-neutralfact-que se suele considerar ligado a otros hechos precedentes. Son, como muy bien replica Pierre Brunel, los excesos del pseudocientificismo que denota la ignorancia de una característica' esencial del hecho comparatista y que es su flexibilidad $(1989,34)$. 
En efecto, se le ha achacado a la literatura comparada de corte tradicional cierto servílismo respecto de otras ramas del saber: la antropología, la historia, la sociología y el psicoanálisis principalmente. Se la ha tildado también de pura erudición basada en la compilación de datos extralíterarios. Es más, cuando algunos investigadores han querido evitar estos ataques, se han limitado a la mera comparación de textos; - no son pocos los que han reducido la literatura comparada al establecimiento de una lista de semejanzas y diferencias entre dos o más textos. Este objetivo no es el idóneo, como tampoco el ataque despiadado de quienes se han limitado a destruir sín ánimo de orientar la investigación comparada. Esto es precisamente a lo que Millán Alba se refería cuando evocaba la proposición que Julia Kristeva hacía del término transposición. Después de abordar los procesos arriba mencionados-metonimia y metáfora-Kristeva aludía a la necesidad de explicar un tercer proceso-Ia intertextualidad-que consiste en el paso de un sistema de signos a otro. Dada la importancia del párrafo, no me resisto a copiarlo

Que le déplacement et la condensation s'y conjuguent Pout l'effecluer, n'épuise pas l'ensemble de l'opération. Il s 'y ajoule une transformation de la posilion thétique: la destruction de l'ancienne et la formation d'une autTe. Le'noveau système signifiant peut être produit dans le mềme matériau signifiant: par exemple, dans le langage, le passage peut s'effectuer de la scène carnavalesque au texte écrlt (... ) Le terme d'intertextualité désigne celte transposition d'un (ou de plusieurs) systéme(s) de signes en un autre; mais puisque ce terme a été souvent entendu dans le sens banal de 'critique des sources' d'un texte, nous luipréférerons celui de transposition, qui a l'avantage de préciser que le passage d'un système signifiant $\grave{a}$ un autre exige une nouvelle articulation du thétique-de la positionnalité énonciative et dénotative. Si on admet que toute pratique signifiame est un champ de trans. positions de divers systemes signifiants (une inter-textualité), on comprend que son 'lieu' d'énonciation et son 'objet' dénoté ne sontjamais uniques, pleins et identiques, à eux-mêmes, mais toujours pluriels, éclatés, susceptibles de modèles tabulaires. La polysémie apparaft donc aussi comme le résultat d'une polyvalence sémiorique, d'une appartenance divers systemes sémiotiques. (1974,59-60)

En la línea de cuanto llevamos dicho, salta a la vista la importancia de este término por cuanto facilita la apertura del texto que la crítica partidaria de un estructuralismo a ultranza sẹ obstinaḅa en considerar clausurado en sí mismo (Millán Alba 1990, 15).

Quizá el error de unos y otros resida en un planteamiento considerado apodíctico pero que no lo es tanto. Muchos comparatistas han creído que'la 
literatura comparada consiste en comparar. Nada más ajeno a la realidad. Unos han cometido una confusión de tipo metonímico y han extendido al substantivo la calificación del adjetivo; otros han buscado la esencia de la literatura comparada en la historia y, sin percatarse, han desvinculado a la ciencia literaria de su forma histórica. Entre unos y otros han ofrecido así un flanco fácil a quienes, sin saber literatura comparada, se percataban-era lo más fácil-del desacierto efectuado por comparatistas inexperimentados. Si la literatura comparada es lo más opuesto a algo, este algo es comparar.

Lo ha visto con lucidez Laurent Jenny al llamar la atención sobre la adición confusa y misteriosa de influencias en que habían caído aquellos comparatistás. Frente a este enfoque inexacto, el crítico definía la intertextualidad como «e travail de transformation et d'assimilation de plusieurs textes opéré par un texte centreur qui garde le leadership du sens ('La stratégie de la forme'), s ${ }^{9}$ Millán Alba elogia los términos de transformación y asimilación aquí utilizados. No le falta razón. El estudio de la intertextualidad existente en un texto receptor nos permite observarcon claridad que respecto a otros textos-1lamémoslos emisores o precedentes, poco importa para el caso-el texto sometido a estudio es único. Utilizando las palabras de Millán Alba, diremos que es singular, esto es, da cuenta por sí mismo del «proceso de encarnación en lo formal en el que consiste todo acto de literatura» (]990, 13).

Este proceso al que alude Jenny es de orden semántico y se refiere a los mecanismos de producción textual, señalados por Millán Alba-retórica, poética, etc.-quien concibe la intertextualidad como uno de ellos. Aquí es preciso hacer un inciso harto interesante. Dejando al margen la idea, de nuevo esgrimida, pero ahora de manera implícita, de que la intertextualidad sea o no un proceso y, por ende, una función, conviene considerarla como una categoría literaria que nos permite acceder a los auténticos procesos de redistribución posibles del hipotexto en el hipertexto (escisión, concisión, condensación, extensión, expansión, amplificación, etc.) expuestos por Genette.

\section{Superación pragmática de las extrapolaciones}

La literatura es libre, nada la puede coaccionar hasta el extremo porque en ese preciso instante dejará de ser literatura. Hay algo más que muchos críticos no quieren ver: el trabajo mismo de lo infinito, como 10 denomina Víctor Rugo, atravesando el cerebro humano. De nada sirve querer recluir en fónnulas todos y cada uno de los procesos: siempre habrá alguno que se nos escape aquí, dos ahí, tres allá: sería como querer poner puertas al campo. La literatura comparada, llevada hasta sus últimas consecuencias, puede legítimamente extender esta concepción de la intertextualidad y superar así los errores de estas extrapolaciones. Su carácter universal no sabe acomodarse a los estrechos marcos de un 
único texto. Por consiguiente, lejos de contentarse con las Limitaciones con que nació la teoría de la intertextualidad, la literatura comparada se revela como la disciplina ideal para desarrollar, sin forzar el objeto de estudio, cuanto habían esbozado las primeras teorías sobre esta categoría. «Si l'on ajoute à ce cas de figure [la lecture d'un texteJ les lectures 'binaires'(deux textes à comparer), les programmes à plusieurs textes et la méthode par 'superpositon' qui méritera en son temps une présentation détaillée, on découvre à ,quel point le mol 'comparaison' recèle de possibilités de lectures» (Pageaux 1994, 18). Asíes, el estudio analítico es indispensable, pero no se da el paso decisivo hasta que no se procede a una síntesis comprehensiva que nos permita llegar hasta el fondo de la cuestión. ${ }^{10}$

Es más, si nos quedamos con el mero repertorio de semejanzas y diferencias o de remodelaciones que un hipotexto ha sufrido en el hipertexto. no habremos avanzado en el terreno de la literatura comparada. como tampoco en la auténtica comprensión del texto sometido a estudio. El comparatista debe ir siempre hasta el fondo de la cuestión, para lo cual requiere la confrontación de al menos dos textos-a menudo muchos más---en diferentes lenguas, de diversas mentalidades en diferentes áreas culturales, de la diversidad de lemas, géneros y poéticas para poder comprender en toda su profundidad tal o cual autor o texto determinado. De ahí que me parezca más acertado, gracias a su moderaci6n,el comentario de Michael Riffaterre: «el Íntertexto es la percepción, por el lector, de las relaciones entre una obra y otras que la han precedido o seguido.»11 De ello se deduce algo de importancia capital para la literatura comparada, puesto que este mismo crítico concibe que la intertexmalidad es el mecanismo propio de una lectura auténticamente literaria: mientras una lectura lineal, común a los textos literarios y no literarios, no aporta sino el sentido, la intertextualidad produce la significación. La intertextualidad puede aportar. y de hecho aporta, un gran cúmulo de nuevas posibilidades a la literatura comparada, pero no es ni el único procedimiento del que se sirve ni tampoco su objetivo.

No me resisto a transcribir un texto de Víctor Hugo. L'art et la science, que puede servimos de pauta para comprender el vaivén que se da entre las teorías arriba esgrimidas y el carácter reaccionario de la literatura frente a las ciencias empíricas: icuántas veces el estudio de la literatura se ha querido resumir en una serie de leyes que la desvirtúan de su esencia! Si se comprende esto, se podrán sopesar los pros y los contrás, reconsiderar algunas teorías sobre la intertextualidad, determinar sus aciertos y desechar sus errores.

Flot surfloto vague après vague, écume derrière écume, mouvement, puis mouvement. L'Iliade s'éloigne, le Romancero arrive: la Bible s'enfonce, le eoran surgir; après l'aquilon Pindare vient l'ouragan Dante. L'étemelle poésie se répete+elle? Non. Elle est la même et elle 
est autre. Mème souffle, autre bruit.

Prenez-vous le Cid pour un plagiaire d'Ajax? Prenez-vous Charlemagne pour un copiste d'Agamemnon?-"Rien de nouveau sous le soleil." - "Votre nouveau est du vieux qui revient."- - etc, ere. Oh! Le bizarre procédé de critique! Done l'art n'est qu'une série de eontrefaçons! Thersiste a un voleu'. Falstaff. Oreste a un singe, Hamlet. L'Hippogriffe est le geai de Pégase. Tous ces poètes! Un tas de tire-laine. On s'entre-pille, voilà tout. L'inspiratíon se complique de filouterie. Cervantès détrousse Apulée. Alceste escroque Timon d'Athènes. Le boís Sminthée est la fôret de Bondy. D'où sort la main de Shakespeare? De la poche d'Eschyle.

Non! Ni décadence, ni renaissance, ní plagiat, ni répétition, ni redite. Identité de coeur, différence d'esprit: tout est là: Chaque gTand artiste, nous l'avons dit ailleurs, refrappe l'art à son ímage. Hamlet. e'es! Oreste à l'effigie de Shakespeare. Figaro, e'est Seapin à l'effigie de Beaumarehais. Grangousier, e'es! Silène à l'effigie de Rabelais.

Tout recommenee avec le nouveau poète, et en méme temps rien n'est interrompu. Chaque nouveau génie est abíme. Pourtant ilya tradition. Tradition de gouffree à gouffre, e'est là, dans l'art comme dans le firmament, le mystère; et les génies eommuniquent par leurs effluves comme les astres. Qu'ont-ils de commun? Rien. Tout. (1985, 33-34)

Dentro de la praxis de la intenextualidad-huelga decir que no hay auténtica praxis sin teoría ní teoría sín praxis-podemos ver que la intertextualidad puede ayudarnos, y de hecho nos ayuda, a comprender mejor la peri,odización comparada (épocas, movimientos, escuelas, temas, mitos, tipos, formas, géneros, poéticas y tantos otros aspectos inherentes a la literatura).

La intertextualidad se puede dirigir según una metodología general o particular. Cabe, como sugiere Broich, limitarla al estudio de los casos en los que un escritor no solamente utiliza textos de otros escritores. sino que además los nombra explícitamente. ${ }^{12}$ La ventaja de este método es evidente: excluye toda posibilidad de error, puesto que el lector lee las referencias a otros textos como parte primordial de la estrategia del texto que tiene bajo sus ojos; más aún: dicho autor es consciente de que el lector también cuenta con este modo de lectura. El diálogo se realiza entonces no sólo entre otros textos y el autor que los recibe sino también entre éste último y el lector.

El objetivo es sano siempre y cuando se mantenga este principio. Sin embargo, , hay que decir que dicha metodología comparatista puede adolecer, llevada hasta sus últimas consecuencias, de la amenaza que antaño rondaba continuamente en tomo a los hábitos comparatistas: el positívismo a ultranza. Sería entonces dañino 
que, procurando evitar el paradójico idealismo al que se habían visto abocados los post-estrocturalistas debido asu método inmanente, fuéramos a parar en el extremo opuesto y redujésemos la presencia de un texto determinado sólo a las ocasiones contadas en las que el autor nos la indica positivamente. Los peligros son numerosos. En primer lugar porque los estudios de recepción, que tanto han aportado últimamente a la literatura comparada, serían obviados (y, con ellos, el bagaje sociocultural que informa a tal o cual autor incluso cuando éste no sea consciente de ello). En segundo lugar porque también forma parte de la estrategia del texto (o, en este caso del autor), elaborar otras ideas aparecidas en textos precedentes sin voluntad de nombrarlos, en unas ocasiones con fines aviesos, en otras, con fines más discretos y respetables.

Dicho con pocas palabras, la intertextualidad, sea o no explicita, existe; y también la libertad del autor. Sólo daré aquí un ejemplo: Hernani, de Víctor Hugo. Una hora antes del matrimonio entre Ruy Gómez de Silva y Doña Sol, un peregrino llega al castillo donde reside el favorito del rey. Está de paso, según dice, hacia el Pilar de Zaragoza donde ha de cumplir una promesa a la Virgen. siguiendo las leyes dictadas por Alfonso X el Sabio en las Siete Partidas, Ruy Gómez de Silva no duda en darle hospedaje. Es más, también de acuerdo con la costumbre, se compromete a no inquirir sobre su identidad. ${ }^{13}$ Ya dentro de la sala principal donde se encuentran los retratos de sus ilustres antepasados de la casa de Silva, un concurso de circunstancias hace que Hernani se quede a solas con Doña Sol. Tras poner en claro el malentendido (Hemani pensaba que ella le había sido infiel, pero ignoraba su decisión de darse muerte en el momento mismo de su matrimonio con Ruy G6mez de Silva), vuelven los arrebatos de amor que encontrábamos al principio de la obra: De modo súbito, irrumpe de nuevo el valido del rey y se admira, sin dar crédito a sus ojos, al descubrir a su prometida en brazos del supuesto peregrino. Reaparecen las imprecaciones desaforadas ante el honor ofendido. Cuando Hernani se dispone a explicar la razón de todo, Ruy Gómez le corta la palabra y responde con vehemencia dirigiéndose a sus antepasados: «Cree / sin duda que mi brazo va a derramar sangre en mi castillo, I que quizás mi corazón esconde en sus tempestades / algún tipo de venganza, hermana del festín de las siete cabezas, I os dirá que es un proscrito, os dirá I que se hablará de Silva como se habló de Lara.»14 No puede menos que extrañar esta alusión a un hipotexto del corpus romarlceril. Pero una nota a un poema titulado «Romance mauresque» de Las orientales explica esta recurrencia: en dicha aclaración, Hugo exponía su teoría sobre la existencia de un Romancero «moro.» Lo hacía con motivo de la venganza que Mudarra tomó sobre Ruy Velázquez, el tío de los infantes de Lara, degollados por siete reyes musulmanes en la sierra de Arabíana por orden de Almanzor. ${ }^{15}$ La atmósfera, pues, no puede ser más árabe; tanto más dado el protagonismo del «hijo de la renegada.» Hasta este momento de la obra, 
no se había hecho referencia alguna a ese romancero moro, y sin embargo el lector conocedor de Hugo sabía que tarde o temprano acabaría por aparecer alguna referencia al ambiente moro. El autor, en el prólogo, se había limitado a decir que el Romancero era la verdadera clave para comprender Remani. Pero hasta ahora s610 había aparecido la cara cespañola»-que él también denomina gótica-de los romances: era la referencia al Cid como vasallo del rey Sancho de Castilla en su asedio a la ciudad de Zamora, entonces bastión de la resistencia leonesa; era también la referencia a Bernardo del Carpio, quien suplicaba al rey Alfonso el Casto la libertad de su padre y se enfrentaba a Roldán en Roncesvalles. La pieza Hemani habría quedado coja si no saliera a relucir una parte tan importante como era el ciclo de la frontera andaluza o el de los romances «moros,» tal y como los denominaban Simonde de Sismondi, otros historiadores, Abel Hugo, su hermano Víctor y el imaginario francés sobre la España del momento. Las modificaciones operadas por Hugo son aquí menores; además de poner en sordina su fuente de inspiración, el autor se limita a emplear el material sólo en funci6n de la prosodia, el ritmo y la rima. Con lo cual el color local queda todavía más patente, así como la sensaci6n de pervivencia, en la memoria colectiva de la España medieval, de elementos íntimamente ligados a la Reconquista y, por ende, a los sucesos de la frontera andaluza. En definitiva, se trata de la cristalización del hipotexto de una «Ilíada árabe» de la cual no dice nada el autor en toda la obra pero que aparece en un cúmulo de estudios y obras anteriores y posteriores: el prefacio de Cromwell, Nuéstra Señora de París, e incluso otro hipotexto que dio nacimiento a toda esta definición del Romancero: la traducción que Creuzé de Lesser diera de los romances españoles; en su estudio preliminar los denominaba «extraña Ilíada sin Homero,» traducci6n que Víctor Hugo utilizó pero que nunca llegó a mentar. ${ }^{16}$ Gracias a estos hipotextos comprendemos el hipertexto de Hernani en toda su profundidad; accedemos mejor a determinados textos que hasta ahora se obstinaban en quedar herméticamente cerrados al crítico. Hay intertextualidad explícita e intertextuaHdad implícita.

\section{Fallen Angels}

Sería ilusorio querer trazar aquí un resumen de la tradici6n que remonta a los más ancestrales mitos hindúes y egipcios sobre la creación. También sería pretencioso intentar las reminiscencias que dichas corrientes dejaron en la literatura occidental; pero en este caso es lícito esbozar al menos algunos de los parámetros indispensables. A lo largo de este corto recorrido es fácil ver la importancia que tiene la transmisión de textos que nosotros denominamos clásicos y su consiguiente recepción por las lenguas y literaturas modernas que aquí nos interesan.

Un mito que siempre ha obsesionado a los grandes escritores es el del origen del mundo y de sus primeros vagidos. El que ya Ovidio abordara este tema en sus 
Metamorfosis es importante por diversos motivos. Entre otros, por cuanto de esta fuente beberían posterionnente Dante y Milton. ${ }^{17}$ Pero, como bien es sabido, al mentar a estos dos últimos escritores estamos haciendo implícitamente alusi6n a otra fuente común; es más, la categoría de la intertextualidad nos pennite ampliar esta investigacion a los mecanismos de creacĺón que presuponen, por una serie de motivos de carácter. formal, la elección de un género determinado-poesía, y preferentemente épica. En efecto, toda una corriente que remonta a los primeros siglos del cristianismo incide sin cesar en las teorías cosmogónicas y la dialéctica de fuerzas del bien y del mal. Piénsese en Orígenes y su Selecta in Genesim. En definitiva, siguiendo esta corriente ascendente, llegamos a la Biblia y sus derivados heterodoxos.

Fuente inagotable de inspiraciones poéticas, la Biblia irrumpe por doquier, ya sea de manera abiert<t o solapada, por ejemplo en Shakespeare; de allí que se haya podido decir que «it is almost a truism that the English Bible was a source-book fer him-a source-book not so much for plots as for themes and language» (Black 1979, 1). Prácticamente todas las obras del dramaturgo contienen referencias bíblicas: As You like it (Gén. 7), Macbeth (Gén. 11.7, Ef. 6), Hamler (Mat. 5.7. Ef. 5.1-4), Measurefor Measure (Mal. 5.34-36), The Comedy o/Errors (Lue. 15), Henry IV (donde se discierne sin duda alguna la parábola del hijo pródigo), etc. Por no citar sino un ejemplo, pensemos en Titus Andronicus donde leemos:, «Now let hot Etna cool in SicHy, ! and be my heart an ever-burning hell ! ! These miseries are more than may be borne.! To weep with them that weep doth ease sorne deal, ! But sorrow f10uted at isdouble death»(I1I,i.241-245). A nadie se le esconde que ese «llorar con los que lloran» de Shakespeare delata la presencia de la parte moral en la que San Pablo exhorta a los cristianos de Roma concretando la caridad que han de vivir entre sí: "Alegraos con los que se alegran, llorad con los que lloran» (Rom. 12.15).

Este entusiasmo por la Biblia crece aún más durante el siglo XVII inglés, alemán y español, „8 para decrecer posterionnente. Será el romanticísmo-desde el místico heterodoxo que fue Blake hasta el viajero infatigable que fue B yron-el que revitalice los principales temas bíblicos y, de modo particular, dos de ellos que cada día adquieren más interés en la crítica literaria: los mitos de Prometeo y del ángel caído. Posterionnente, los diversos movimientos europeos-realismo, prerrafaelismo, naturalismo,ete.-aun sin excluirlos, relegan estos temas a un segundo plano. Si acaso les interesa Prometeo, pero s610 por su significaci6n metaliteraria. Sea como fuere, es cierto que la Biblia dejó de ser un punto de referencia obligado hasta las primeras décadas de este siglo. Quizá porque tras la belle époque todo parecía asemejarse a las catástrofes de Sodoma y Gomorra (no en vano Proust titula así uno de los libros de À la Recherche du temps perdu); de hecho, el presentimiento de las conflagraciones mundiales sugería en la mente de 
no pocos autores reminiscencias bíblicas. Basta leer el Ulysses de Joyce para comprobarlo: Leopold B 100m deambulando por el mercado o durante las horas pasadas en compañía de Stephen Dedalus se comprenden en toda su profundidad teniendo como hipotexto escenas del Nuevo Testamento. Pero otro tanto ocurre con Pygmalion, obra escrita en 1913, prueba evidente de que los escritores de los siglos XVI y XVII, así como los textos a los que hacían referencia, no habían pasado desapercibidos a Bernard Shaw. Recordemos brevemente aquella ocasión en la que Doctor Higgins habla por vez primera con Eliza Doolittle. La reacci6n del profesor al oír el cockney de la joven no se hace esperar: «A woman who utters such depressing and disgusting sounds has no right to ... live. Remember that you are a human being with a soul and the divine gift of articulate speech; that your native language is the language of Shakespeare and Milton and the Bible: and don't sit there croonnig like a bílious pigeon.»

El mito del ángel caído reviste capital importancia para la historia general de la literatura. Como quedó expuesto más arriba, la intertextualidad requiere el estudio de la genología. En efecto, bastaría un rápido recorrido por la historia de la literatura para cerciorarse de que el ángel caído es un tema eminentemente épico, y como tal reaparece en Paradise Lost y en el Romanticismo. Después, el tiempo de la épica ya acabó, con el gran epígono que fue La leyenda de los siglos de Víctor Hugo; 19 pero quizá ha sido la última, al menos en el mundo de la literatura europea. Entre otras razones porque, desde una perspectiva antropológica, como bien. decía Carlos Fuentes, «ya no hay un orden ancestral restaurable ni un universo único en su normalidad» (Guillén 1985,33). También porque, desde una óptica puramente lírica, como decía Lamartine, es muy difícil conservar el «sostenido esfuerzo» durante el gran número de versos que requiere todo canto épico. ${ }^{20} \mathrm{Si}$ acaso quedan retazos en la poesía lírica de las Elegías de Duino de Rilke, donde el ángel adquiere progresivamente un carácter positivo; pensemos también en un Alberti (Sobre los ángeles) o incluso en un Aleixandre (Sombra del paraíso), donde, en poemas como «Destino trágico,» el interlocutor ya no tiene aquellas «alas brillantes» del primer Lucifer sino unas «alas oscuras» que le introducen en la vida intra-marina. ${ }^{21}$ No es éste ya el mito del ángel caído en la época romántica.

Quien dice ángel caído en la época romántica dice simultáneamente rebelión, caída, nostalgia y redención. Pbdrán sumársele más o menos temas adyacentes, pero éstos nunca están ausentes. ${ }^{22}$

La mayoría de los poetas ingleses que «escenifican» esta rebelión tienen como referente textual o hipotexto más cercano Paradise Lostde Milton y, más lejano, la Biblia en sus diferentes versiones. Ejemplo harto elocuente es Blake, quien en Marriage ofHeaven and Hell no duda en recurrir tanto a la sedición infernal como al motivo de los treinta y tres 'años que tradicionalmente se atribuyen a la duración' 
de la vida de Cristo sobre la tierra: «As a new heaven is begun, and it is now thirty-three years since its advent: the Eternal Hell revives» (Blake 1988, 34). Otro ejemplo especialmente interesante es el de Thomas Moore, cuya obra clave en este sentido es The Laves 01 the Angels, publicada en $1823 .{ }^{23}$ Es de notar el epígrafe de este largo poema, compuesto de 1791 versos: «It happened, after the Sons of Men had multiplied in those days, that Daugthers were born to them elegant and beautiful; and when the Angels, the Sons of Heaven, beheld them, they carne enamoured oí them.» Cuando menos, es curioso que este versículo haya sido extraído del libro de Enoch (cap. 7 [6] seco 2, indica el mismo autor). Podía haber sido extraído del Génesis, pues entonces circulaba con gran éxito, especialmente en su descripción del diluvio, mito literario por excelencia en relación con el ángel caido. ${ }^{24}$. Es lo que hizo Byron en Heaven and Earth. A Myslery, quien incluye como epígrafe el texto siguiente: «Founded on the following passage in Genesis, chapo 7: 'And it carne to pass .... that the Sons oí God saw the Daughters of Meo that they were fair; and they took them wi ves of aH which they chose'» $(1964,545)$. Estos dos versículos (1 y 2 del capítulo 6), son el prólogo al relato del diluvio y han suscitado no pocas discusiones debido a su estado fragmentario. No cabe la menor duda de que la intención del autor es poner de relieve la malicia de la humanidad en progresión creciente. Problema más acucíante aun es la interpretación de los sintagmas «hijos de Dios» y las «hijas de los hombres.» La ortodoxia católica ve en los «hijos de Dios» a los descendientes de la raza elegida de Set o setitas y en las «hijas de los hombres» a las descendientes de la raza de Caín o cainitas. Por otro lado, la unión es presentada como repulsiva poligamia (Biblia 1964, 15-17). En circunstancias normales, el desarrollo de una obra que se sirviera de este texto debería contener este tipo de relaciones. No fue así. En primer lugar por razones de orden doctrinal quizá desconocidas o eludidas por Byron; en segundo lugar, y esto es lo que nos interesa, porque la transposición de textos de la que hablaba Kristeva se ha producido en toda su eficacia. Para Byron, como consecuencia de su conocimiento de Swedenborg, Blake y Moore, entre otros, los «hijos de Dios» son los ángeles. Ángeles caídos, más precisamente, como se observa al final de esta obra: los ángeles Samiasa y Azazie1.25 A pesar de las amonestaciones del arcángel Rafael, parten en busca de otros mundos en compañía de las mujeres Anah y Aholibamah (Byron 1964, iii.558) momentos antes de que el diluvio caiga sobre la tierra: estamos ante la prolongación de un mito que tiene sus raíces en la Biblia y las sucesivas elaboraciones de los diferentes escritores a lo largo de la literatura. Pero aún hay más. Byron no 5610 ha tenido en mente el epígrafe indicado: junto a éste último añade un verso de Coleridge: «And woman wailing for her demon lover.» Lo que estas palabras hayan podido sugerir en la composición de Heaven and Earth salta a la vista si 10 conjugamos con el epígrafe arriba citado de Thomas 
Moore (Enoch 7). Estos amores en los que las mujeres, aquí Anah y Aholibamah, gimen por el amante demoníaco-una virtualidad del ángel caído--ponen de manifiesto que Byron no solamente constituía su obra con el referente del Génesis, sino también con el de Enoch. Prueba harto sobrada es que este libro apócrifo aparece en un soliloquio de lafet: «God hath proclaim'd the destiny of earth; I My father's ark of safety hath announced it; / The very demons shriek it from their caves; I The scroll of Enoch prophesied it long / In silent books, which, in their silence, say I More to the mind than thunder to the ear» (1964. iii.552). Curiosa relación, sobre todo vista desde la mentalidad contemporánea, entre seres angélicos y seres humanos. Pero estamos èn pleno romanticismo, época y movimientos extremadamente proclives a este arquetipo del inconsCiente colectivo debido a sus connotaciones teosóficas y antropológicas. Además de los múltiples estudios que entonces se hicieran en Francia, s610 señalaré que estas producciones venidas del norte hallaron un campo abonado en este último país para la elaboración francesa del mito del ángel caído. Dado que ya lo he podido estudiar detenidamente en Tristán y su ángel, no haré aquí sino limitarme a recordar algunas de las obras señeras que recogen este mito y lo adaptan según la mentalidad literaria y antropológica del momento: Le Répentir de Elzéar de Sabran (1817), La Divine épopée de Alexandre Soumet (1840), varios poemas de las Méditations de Alphonse de Lamartine, así como su largo poema La Chute d'un ange (1836-1837), varios poemas de Alfred de Vigny-especialmente «Le Déluge, » «Éloa» y «Satan»--y el libro de Víctor Hugo, publicado a título póstumo bajo el título de La Fin de Satan (1886).

Decía que se le podrían sumar otros aspectos adyacentes; uno es el prometeico. ¿Qué significa este titán sino la rebelión contra los dioses en favor de los hombres? No podía faltar, por ejemplo, en Byron, cuyo «Prometheus» canta al gigante que no pudo soportar ver con sus «immortal eyes $I$ me sufferíngs of mortality» $(1964,98)$. Por otra parte, constatamos que, además de las fuentes clásicas, esta pieza de Byron ha sido bien acogida entre los románticos franceses. Me detendré sólo en unos versos, aquéllos en los que el viajero de Missolonghi describía la raz6n de la rebelión y la tortura a la que Prometeo fue sometido: «Titan! to thee the strife was given I Between the suffering and the will, I Whích torture where they cannot kili: I And the inexorable HeavenJ And the deaftyranny of Fate, I The ruling principie of Hate; I Which for its pleasure doth create I The things it may annihilate, / Refused thee even the boon to die» (98).

La intertextualidad puede verificarse en diversas obras de un mismo autor. Precisamente el mito prometeico nos permite hacerlo en relación con el otro arriba enunciado, lo cual no viene sino a corroborar la íntima relación existente entre ambos en la época romántica y las ricas interpretaciones metaliterarias a las que se presta. Así, los motivos principales de la tortura sufrida por el titán «The rack, 
the vulture, and the chain» («Prometheus» 1964, 98), reaparecen en otro poema de tema bíblico pero sometidos a una ligera modificación según la ley de flexibilidad. Recordemos a Jafet advirtiendo al ángel Azaziel del peligro al que se expone si se rebela a la voluntad de Dios: «Sorne clouds sweep on as vultures for lheir prey, I While others, fix'd as rocks, await the word I At which their wrathful vials shall bepour'd.» De nada sirve esta amenaza pues el ángel invita inmediatamente a la mujer Anah a seguir su destino: «Come, Anah! quit tmB chaos-founded prison» (Heaven and Eanh 1964, 558).

Más interesante, y habitualmente más difícil, es constatar la aparición de textos extranjeros. Un ejemplo elocuente es el del Romancero español, que tantas composiciones inspirara entre los poetas románticos alemanes, franceses e ingleses. Entre estos últimos, recordemos al obispo Thomas Percy y sus Reliques o/ Ancient English Poetry (1765); a Savage Landor, Lord Holland; a Robert SDuthey; por ejemplo en su Chronicle oithe Cid y su largo poema Roderick, the Last 01 the Goths (1814); a Walter Scott; su yerno J.-O. Lockhart, quien tradujo en 1823 cincuenta y tres romances en sus Ancient Spanish Ballads; a John Bowring con un interesante estudio titulado Spanish Romances y su traducción tituladaAncient Poetry and Romances oiSpain Selected and Translated, y al mismo B yron quien, aunque sólo esbozó algunas ideas en su poema inconcluso Don Juan (13.11), no dudó en adaptar el romance del moro que perdió el sitio de Alhama. Este poema, A Very Mournful Bailad on the Siege and Conquest oiAlhama, puede servir, y mucho, para los estudios de intertextualidad como lectura comparatista. ${ }^{26}$

De igual manera, puede observarse la acogida de que Byron gozara en Francia desde la segunda década del siglo XIX y que más abajo veremos en lo que a Víctor Rugo atañe. Delécluze, Mme. de Rémusat, Chastopalli, Vanderbourg y Arrnand de Ponmartin dieron rendida cuenta de ello en vida del poeta. Mayor fue aún el ruido que provocó su óbito, momento crucial que puso de manifiesto hasta qué punto era leído en Francia. Los artículos publicados en la prensa del momento son una prueba fehaciente. Así, Roch compuso una quinta «Helléníde,» una especie de "poema ditirámbico sobre la muerte de lord Byron» que publicó en $L a$ Pandore los días 29 de mayo y 1 de junio de 1924. Movido por la misma veneración, el gran traductor que fuera Damas Hinard publicaba sus Chants sur lord Byron en la misma revista con fecha del 20 de diciembre del mismo año. Y a principios del siguiente salía a la luz un libro de Mme. Louise Swanton-Belloc titulado Lord Byron. ${ }^{27}$ A estas primeras reacciones surgidas como consecuencia de la desaparición del poeta hay que sumar los comentarios de Mme. de Lamartine, publicados por su hijo en Le Manuscrit de ma mére el 4 de enero de 1825, el poema que le dedicara Privat d' Anglemont bajo el título de «La mort de lord Byron» en Pandore, el 15 de marzo de 1825, e incluido en sus Odas de juventud, a los que es preciso sumar los artículos anónimos aparecidos en Le Globe (12 de 
octubre de 1824) y en Le Constitutionnel (19 de enero de 1825). En definitiva, no cabe duda alguna de que la pérdida de Byroo supuso en Francia una «calamidad pública,) por utilizar la expresión de Dumas padre en sus Mémoires (18521854). 28

Todo esto da pie para imaginar el impacto que pudo tener la obra del poeta inglés en Víctor Hugo. Además de otras composiciones, no está de más ver cómo funciona el texto arriba citado en alguna composición de éste último; la constatación de este hecho puede ayudarnos a comprender mejor la categoría de la intertextualidad. Haciendo uso de lo que Pierre Brunel ha denominado la ley de flexibilidad $(1989,34)$, podemos observar que Hugo toma varias ideas de los versos del poema «Prometheus;» pero lo hace adaptando según su universo imaginario. En efecto, de la misma manera que Montaigne hablaba de la «flexibilidad de nuestra capacidad inventiva para sacar partido a todo tipo de ensoñaciones,» Hugo defonna relativamente el texto de Byron sacándole el mayor partido que requiere su ideal del hombre nuevo. Reincidiendo en su idea del progreso al que aspiran los hombres desligados de los dioses, Hugo ve en el titán el punto de apoyo para salir de la indigencia a la que las falsas divinid.ades los han sometido. Prometeo simboliza por lo tanto el impulso soberano que «hace vivir a aquéllos a quienes el destino ha hecho nacer» y procura arrojar «por siempre el odio vil al Tártaro» («Le Vautour.» En Dieu. 1984, 1046-1047). El - destino-Fate-al que Byron aludía queda así eliminado y los hombres pueden, finalmente, aspirar al amor, única fuerza capaz de conjurar el odio-Hate - que los divide. De esta manera, lo que en Byron era compasi6n por el titán sufriente se transfonna en el poema francés en un alegato más para apoyar la idea obsesiva del autor sobre la redención y consiguiente libertad a la que aspira el nuevo hombre; el mismo Hugo expone ampliamente este anhelo en los dos poemas comprendidos en el capítulo titulado «Vingtieme siècle» de La Légende des siècles. En ellos el hombre ya aparece precisamente sin cadenas, es el Prometeo desencadenado de Shelley, que sube porque ya nada 10 ata a la roca donde soportaba el martirio descrito por Byron. Prometeo aparece pues, en la obra de Hugo, como «el símbolo de los tonnentos que renacen incesantemente queriendo devorar por siempre el corazón del hombre que ha tomado el camino de la ciencia» (Roos 1958,44). De hecho, en el poema «Pleine mer» y en «Pleine cíel,» Hugo nos presenta al hombre que, gracias al verdadero progreso, concreción del Prometeo de los autores ingleses, ha conseguido deshacerse de cuanto aparecía en el poema de Byron: todo tipo de trabas (《<los dioses eran los muros»), rencores («Todo se fue de una vez, y los furores, los odios»), sistemas ( «El derecho de los reyes, los falsos dioses judíos,» que no son sino «criaturas para Dios») y lo lanza hacia arriba, «orgulloso, arrancando el barro de su cadena;» el «esfuerzo del cautivo» Prometeo y su «rabia santa» han conseguido que el hombre «se trans- 
fonule] en ángel y, rompiendo sus ataduras» se eleve hasta los cielos: ,en definitiva, en ese mismo instante, «Entonces, ¡la muerte será inútil!» (1994, 295-319).

Salta a la vista que la obsesión hugoliana por la recuperación del hombre pasa por la ansiada síntesis de Prometeo y el ángel caído, la misma que, siguiendo en gran medida lateosofía de Swedenborg, promovieron tantos románticos alemanes e ingleses. El autor francés, tenía conciencia de los deseos de los poetas que le habían precedido; en su texto-hipertexto en este caso-hay intertextualidad, pero también una reelaboración que en modo alguno depende exclusivamente de los hipotextos: éstos suponen el punto de apoyo para el poeta, influido pero libre, escogiendo entre lo que unos y otros le ofrecen, desarrolle el mito de acuerdo con su propio mundo imaginario.

\section{Notas}

I Sobre los cinco tipos de relaciones transtextuales y sus diferentes subdivisiones (íntertextualidad [cita, plagio o alusión); paratextualidad [o relación]; metatextualidad [o comentario\}, architextualidad e hipertextualidad), $c f$. Genette (1982,8-14).

${ }^{2}$ «Le concept d'intertextualité a provoqué un vif intéret du fait que les procédures qu'i1 impliquait semblaient pouvoir servir de rechange méthod'ologique la théorie des influences sur laquelle se fondaient, pour I'essentiel, les recherches de la littérature comparée.;; 3 «Le dialogue, au sens, étroit du terme, ne constitue, bien entendu, qu'une des formes, des plus importantes, il est vrai, de I'interaction verbale. Mais on peut comprendre le mot 'dialogue' dans un sens élargi, c'est-à-dire non seulement comme I'échange à haute voix et impliquiant des individus placés face à face, mais tout échange verbal, de qúelque type gu'il soit.y

${ }^{4}$ A pesar de estas discrepancias, sí es verdad lo que Millán Alba indica sobre el carácter eminentemente literario de la intertextualidad. De ahí que esta categoría nos interese: porque nos permite estudiar el texto con un talante exclusivamente literario. Ahora bien, esto no significa rehusar la aportación, indispensable y simultánea, de otras ciencias que nos ayudan a comprender mejor la literatura. MiUán Alba enuncia un procedimiento que me parece discutible al extrapolar un determinado enfoque a todo el quehacer científico. No le falta razón al lamentarse de «la excesiva preocupación por dotar a ambas disciplinas [la historia de la literatura y la literatura comparada] de un statu [sic] 'científico,' haciendo derivar este estado de aspectos epistemológicos o metodologías críticas e históricas, y no de la naturaleza del objeto sometido a examen.» Hasta aquí estamos completamente de acuerdo, pero la consecuencia que saca de ello adolece de cierta parcialidad: «Así, ya desde el principio, el acento se desplazó del 'qué' al 'cómo,' a los distintos 'c6mos' históricos y. metodológicos, como si el ideal de estas metodologías fuese el de la producción y explicación en serie» [sicJ. Es difícil juzgar con ecuanimidad el trabajo que otros han comenzado en circunstancias muy difíciles y en nada parecidas a las actuales. y concluye, ahora sí, con indudable acierto: «Una de las consecuencias de esta actitud ha sido la de considerar el texto literario--y en general, el fenómeno estético--como un puro epifenómeno de otras disciplinas» $(1990,13)$. 
5 El lector habrá reconocido la definición de Julia Kristeva según la cual el texto es un «mosaïque de c.itations.»

6 Constato con agrado que Millán Albadice algo semejante respecto de estas desviaciones: «El desarrollo ulterior de la [intertextualidad] ha ido desplazando el acento desde dicho sistema de signos a los espacios en los que encuentra su razón de ser: sociales y pulsionales, a los que pueden añadirse las superestructuras histórico-económicas. las infraestructuras arquetipales de la antropología y las mismas estructuras lingüísticas ( . . . ) Dicho corrimiento del-acento a los distintos espacios que acabo de enumerar y que funcionan-analógicamente-a modo de textos emisores, muestra una evidente similitud estructural con la antigua 'crítica de fuentes's $(1990,17)$.

7 «Insistons d'ailleurs sur ceci, car l'émulation des esprits c'est la vie du beau. ô poètes: le premier rang est tojours libre. Écartons tout ce qui peut déconcerter les audaces et cásser les ailes; l'art est un courage» ( «'A'Art et la science» 1985,35).

8 Nier que les génies survenants puissent étre les pairs des génies antérieurs, ce serait nier la puissance de Dieu. Oui. el nous revenons souvent, et nous reviendrons encore sur cet encouragement nécessaire, stimulation c'est presque création; ouí, ces génies qU'Oli ne dépasse point, on peut les égaler. Comment? En étant autre» (35).

9 Cabe hacer un reparo. Al identificar la íntertextualidad con un trabajo, vemos que Jenny comete el mismo error de Millán Alba. Ambos confunden una categoría con su funcionalidad. y otro tanto comete éste último crítico al afirmar, comentando la definición de Jenny, que se podría substituir las «voces» de transformación y asimilación por la de estructuración. No es sino una consecuencia del mismo desacierto: la intertextualidad, al no «funcionar» o «trabajar,» no puede estructurar.

$10 \ll$ Conviene reparar en el doble movimiento operativo subyacente a las distintas doctrinas enumeradas [psicoanálisis lacaniano. teoría durandiana, etc.]. De un lado, se observa que los movimientos posteriores son 'explicados' por los anteriores. en una trayectoria inversa y regresiva que va de lo último a lo pretendidamente primero; asr, cada movimiento posterior es automáticamente congelado en su dinamicidad y retrotraído al anterior. Cabe, sencillamente. preguntarse si este tipo de conocimiento que tiene en su base una concepción analítica regresiva, se adecua a la estructura propia de la obra de arte, que es eminentemente sintética y tendencialmente progresivas (Millán Alba 1990, 17).

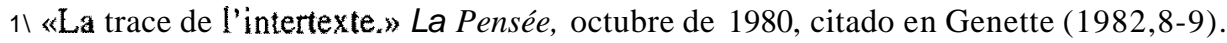
Cf. también «La syllepse intertextuelle.»Poétique 40 (1979). La Production du texte. (París: Éditions du Seuil) y Sémiotique de la poésie. (París: Éditíons du Seuil, 1982).

12 «I shall try to limit the term to those cases where a writer does not only use other texts, but refers to them. This concept implies, ideally, the following process of communication: an author refers to other texts within his own text expecting his readers to understand these references as part oC the strategy of his text» (1989. 120).

13 Son elementos que sin duda Hugo ha tornado de romances donde aparecen estos topoi: el del Cid en su peregrinación a Santiago de Compostela (<<Celebradas ya las bodas a do 1a corte yacía ... »), el de Gaiferos vengador (<<Vámonos-dijo-, mi tío, en París esa ciudad ... ») y el del regreso del hijo de Carlomagno ( «De Mérida sale el palmero, de Mérida esa ciudad....»).

I4 «... Voyez-vous? il veut parler. I'infâme! / Mais. mieux eneor que moi, vous lisez 
dans son âme / Oh! ne I'écoutez pas! e'est un fourbe! Il prévoit / Que mon bras va sans doute ensanglanter mon toit. / Que peut-etre mon coeur couve dans ses tempêtes / Quelque vengeance. soeur du festin des sept têtes. I II vous dira qu'íl est proscrit, il vous dira i Qu'on va dire de Silva comme I'on dit Lara, IEt puis qu'il est mon hôte, e puis qu'il est votre hôte.../ Mes aïeux, mes seigneurs voyez: est-ce ma faute? I Jugez entre nous deux!» (1 Thếâtre iii.v.1232).

15 Se encuentra en el famoso romance cuyo incipit es el siguiente: «Ya se salen de Castilla castellanos con gran saña ... » Sin ser un poema de origen árabe. el tema lo es. así como su continuación: el llanto de Gonzalo Gustios, el consuelo que éste encontró en los cuidados que le dispensó la hermana del rey moro, la revelación que tuvo Mudarrillo sobre su origen bastardo y el celebérrimo romance que diera realce a todo el ciclo: «A cazar va Don Rodrigo y aun Don Rodrigo de Lara .... »

${ }^{16} C f$ el estudio que le dedico à este asunto en Victor Hugo y la literatura moderna. Prolegómenos comparatistas a una lectura de Hernani.» Primeras jornadas de literatura comparada y didáctica de la literatura. Ed. Alfredo Rodríguez' López-Vázquez (La Coruña, 1995).

17 En lo que respecta a la importancia de la Biblia en la literatura moderna, doy algunas referencias no incluidas en la bibliografía. Aun con sus errores, el libro de Northrop Frye, The Great Code: The Bible and Literature (New York: Harcourt Braée Jovanovich, 1982) ha supuesto un avance considerable en estos estudios. Además de,los estudios de Henri Brémond, interesa el libro' de' J.-P. Jossua, Pour une étude religieuse de ['éxpérience littéraire (París: Beauchesne, 1985). Desde el punto de vista comparativo, el lector leerá con interés el artículo de Danielle Chauvin sobre «La Bible dans les études comparatistes. Problemes et perspectives.» Bulletin de liaison el d'injormation de la Société française de littérature générale et comparée8 (1990): 11-24. En lo que a Ovidio concierne, conviene señalar el artículo de Jacqueline Duchemin «La Création et le déluge chez Ovide_ Recherche sour les sources grecqueset orientales du mythe.» Mélanges E. Paratore Bolonia (Patron Editore. 1981). Sobre Dante y Milton, $C f$ entre otros. Richard J. Durocher «Dante, Milton, and Art of Visual Speech.» Comparative Literature Studies XXVII. 3 (1990): 157-171.

18 Curiosamente no en Francia, donde el barroco, importado desde Italia y España, toma en este país un cariz erótico-lúdicro. y donde el clasicismo rechaza-con excepción de algunas tragedias clásicas, ninguna de ellas obra de primera fila-la puesta en escena de elementos sobrenaturalesy realistas como los de la Biblía.

19 En nuestra edición de La leyenda de los siglos (Col. «Letras Universales,» Madrid: Cátedra, 1994) estudio este problema sobre la posibilidad de una obra épica en los tiempos modernos.

20 Poe desarrolla detenidamente este problema de raigambre poética en su Principio poético. (Madrid: Alianza Editorial, 1987): 83.

21 Cf Vicente Aleixandre. Sombra del paraíso. Ed. Leopoldo de Luis. Col. «Clásicos Castalia» 71. Madrid: Castalia (1982): 92-94.

22 Respecto del mito del ángel caído en la Europa romántica, permítaseme remitir al lector a una serie de trabajos centrados en las literaturas alemana, inglesa, española y francesa: 1994. «La mujer y el ángel caído: soteriología en la época romántica.»Actas del lX 
Simposio de ta Sociedad Española de literatura Comparada. Eds. Túa Blesa et al. Zaragoza: Universidad de Zaragoza (1994) 1: 235-244. «La recepción francesa del ángel caído en la época romántica,» incluido en el libro Tristán y su ángel. Diez estudios de literatura general comparada. Kassel: Reichenberger, (1995): 99-144. «Síntesis francesa del ángel caído,» Actas del Congreso «La France au carrefour de I'Europe (en prensa, centrado, como el precedente, en la influencia de los románticos alemanes e ingleses en Francia). «L'Ange déchu et la pitié compatissante.» Studifrancesi XXXIX 116, fase. II (1995) maggio-agosto: 285-293. «La Solitude de j'ange déchu à I'époque romantique.» . Revue Luxembourgeoise de littérature Générale et Comparée (en prensa).

${ }^{23}$ No es posible detenernos aquí en la influencia que Moare tuvo en la obra de Lamartine, Vigny y Hugo. Una prueba incontrovertible del conocimiento que Hugo tenía de Moore, está en la reseña que el poeta francés publicara en Le Conservateur littéraire de junio de 1820 sobre LaUa-Rouk.h. o la princesa mongol de Thomas Maore. Es más, en nombre de Nourmahal en su poema «Nounnahal la Rousse» de Les Orientales ha podido tener su origen en uno de los poemas de Lalla-Roukh. Cf. Pierre Albouy en Victor Hugo (1992): 1297 y 1325.

24 Aquí es obligado mentar The World before the Flood. A Poem, in Ten Cantos, de James Montgomery (Landon: Routledge, 1860).

2S Samiasa también responde a los nombres de Semyaza, Semiaza, Shemhazai, Sharnazya y Amezyarak. Era uno de los cabecillas de los ángeles caídos, también aparece en The Zoar. Azaziel es otro nombre del serafín Samiasa. Anah es una nieta de Caín. Cf. Gustav Davidson (1967): 64 y 265.

${ }^{26}$ Para más informaci6n sobre la influencia del Romancero en Europa envío al lector a mi introducción a la reciente edición francesa.

27 La revista Le Globe daba el 3 de febrero de 1825 una ex.tensa reseña finnada por C. R.. es decir, la misma Mme. Rémusat arriba citada. Acerca del libro de Swanton-Belloc y otras manifestaciones literarias de la obra de Byron de esos años el lector podrá encontrar infonnaci6n suplementaria en el artículo que le dedico a «La recepción francesa del ángel caído en la época romántica» en ellíbro anteriormente citado.

${ }^{28}$ Para mayor infonnaci6n, $c f$. Eric Partridge (1968): 110-118, quien a su vez envía al libro de Estève, Byron et le romantisme français (1907).

\section{Obras citadas}

Bakhtine, Mikhail. 1977. Le Marxisme et la Philosophie du Langage. Tr. Marina Yaguello. Paris: Minuit. 1984. Esthetique de la création verbale. Paris: Gallimard.

Barthes, Roland. 1975. Roland Barthes par Roland Barthes. Paris: n.p.

Biblia. 1964. Ed. Evaristo Martín Nieto. Madrid: Paulinas.

Black, James. 1979. «Edified by the Margent,» Shakespeare and the Bible. Calgary: Faculty of Humanities.

Blake, William. 1988. The Complete Poetry and Prose of William Blake. Newly revised edition. Eds. David V. Erdman \& Harold Bloom. New York: Anchor BooksIDoubleday.

- O 1983. Matrimonio del Cielo y el Infierno. Los Cantos de Inocencia. Los Cantos de Experiencia. Madrid: Visor. 
Broich, Ulrich. 1989. «Ways of Marking Intertextuality.»Fiction. Narratologie. TeX!e. Genre. Proceedings 01 the Intemational Comparative Literature Association. $\mathrm{XI}^{\text {h }}$. International Congress. 2 vols. New York, Bern, Frankfurt am Main, Paris: Peter Lang. 119-129.

Brunel, Pierre e Yves Chevrel, eds. 1989. Précis de littérature comparée. Paris: Presses Universitaires de France.

Byron, George Gordon. 1964. The Poetical Works. London: Oxford UP.

Cioranescu, Alejandro. 1964. Principios de literatura comparada. La Laguna: Universidad de la Laguna.

Davidson, Gustav. 1967. A Dictionary 01 Angels. including the Fallen Angels. New York: The Free Press.

Dupriez, Bernard. 1991, 1984. Gradus. Les Procédés liuéraires (Dictionaire). Paris: Christian Bourgois. 10-18.

Genette, Gérard. 1982. Palimpsestes. La Littérature au second degré. Paris: Seuil.

Guillén, Claudio. 1985. Entre lo uno y lo diverso. Introducción a la literatura comparada. Barcelona: Crítica.

Hugo, Víctor. 1984, 1950. La Legende des siècles. Satan. Dieu. Ed. Jacques Truchet «Bibliothèque de la Pléiade.» Paris: Gallimard.

--O 1985a, 1963. Théâtre completde Victor Hugo. Vol. 1, préface par Roland Pumal, notices et notes par J.-J. Thierry ę Josette Mélèze. «Bibliothèque de la Pléiade,» Paris: GaUimard.

- 1985b. «L'Art et la science.» [fragmento de William Shakespeare] Ed. Jean-Marc Uvy-Leblond. Paris, Nice: Hubeet Nyssen \& Anaïs \& Actes Sud.

- -O 1992, 1964. Euvres poétiques. Vol. 1. Ed. Pierre Albouy. «Bibliotheque de la Pléiade.» París: Gallimard.

- - O 1994. La leyenda de los siglos. Losada Goya. «Letras Universales.» Madrid: Cátedra.

Kierkegaard, Sören. 1975, [1841] «Le Concept d'ironie constantment rapporté à Socrate.» 2 vols. Euvres complètes. Tr. Tisseau. Orante.

Krisleva, Julia. 1974. La Rêvolution du langage poétique. L'Avant garde à lafin du XIX' siecle; Lautréamont et Mallarmé. Pans: Seuil.

1978,1969. Sèméiótiquè. Recherches pour une sémanalyse. (Extraits). Paris: SeuiL

Leon Machado, José. 1995. «Intertextualidade e Intratextualidade no Romance de Verglio FelTeira Nome da Terra.» En Dedalus. Revista portuguesa de literatura comparada 5: $297 \cdot 315$.

Losada Gaya, José ManueL 1995. Tristán y su ángel. Diez estudios de literatura comparada. «Problemata Literaria.» Kassel: Reichenberger.

--O Ed. 1998. Le Romancero. «La Salamandre.» Paris: Imprimerie Nationale.

Millán Alba, José Antonio. 1990. «En torno al concepto de intertextualidad.» Europa en España. España en Europa. Actas del Simposio Internacional de Literatura Comparada. Eds. Hugo Dyserinck. Ángel R. Fernández, Enrique Banús \& Kurt Spang. Barcelona: PPU.

Montgomery, James. 1860. «The World before the Flood.»Poems 01James Montgomery. Londan: Routledge. 
Pageaux, Daniei-Henri. 1994. La Littérature comparée. «Cursus: Littérature.» París: Annand Colin.

Partridge, Erie. 1968. The French Romantics' Knowledge of English literature (18201848). New York: Burt Franklín.

Prado, Javier deL 1993. Teoría práctica de la función poética. Poesía siglo XX. «Crítica y estudios literarios:» Madrid: Cátedra.

Roos, Jacques. 1958. Les /dées philosophiques de Victor Hugo. Bal/anche et Victor Hugo. Paris: Nizet.

Seebaeher, Jacques. 1993. Victor Hugo ou le calcule des profand. «Écrivains.» París: Presses Universitaires de France. 